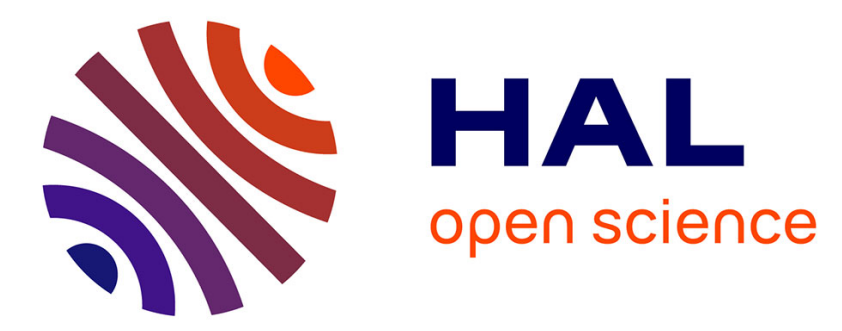

\title{
KEOPS: Kiloparsec Explorer for Optical Planet Search, a direct-imaging optical array at Dome $\mathrm{C}$ of Antarctica
}

Farrokh Vakili, Adrian Belu, Eric Aristidi, Eric Fossat, A. Maillard, Lyu Abe, Karim Agabi, Jean Vernin, Jean Baptiste B Daban, Wilfried Hertmanni, et al.

\section{To cite this version:}

Farrokh Vakili, Adrian Belu, Eric Aristidi, Eric Fossat, A. Maillard, et al.. KEOPS: Kiloparsec Explorer for Optical Planet Search, a direct-imaging optical array at Dome C of Antarctica. SPIE Astronomical Telescopes + Instrumentation, 2004, Glasgow, United Kingdom. pp.1580, 10.1117/12.561498 . hal-02480089

\section{HAL Id: hal-02480089 \\ https://hal.science/hal-02480089}

Submitted on 15 Feb 2020

HAL is a multi-disciplinary open access archive for the deposit and dissemination of scientific research documents, whether they are published or not. The documents may come from teaching and research institutions in France or abroad, or from public or private research centers.
L'archive ouverte pluridisciplinaire HAL, est destinée au dépôt et à la diffusion de documents scientifiques de niveau recherche, publiés ou non, émanant des établissements d'enseignement et de recherche français ou étrangers, des laboratoires publics ou privés. 


\title{
KEOPS: Kiloparsec Explorer for Optical Planet Search, a direct-imaging Optical Array at Dome $C$ of Antarctica
}

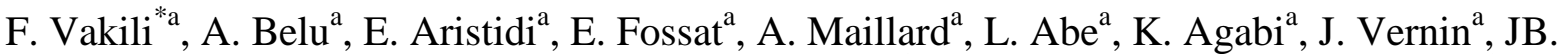 \\ Daban $^{\text {a }}$, W. Hertmanni ${ }^{\mathrm{a}}$, F.X. Schmider ${ }^{\mathrm{a}}$, P. Assus ${ }^{\mathrm{b}}$, V. Coudé du Foresto ${ }^{\mathrm{c}}$, M. Swain ${ }^{\mathrm{d}}$ \\ ${ }^{a}$ Laboratoire Universitaire d'Astrophysique de Nice, Parc Valrose, 06100 Nice, France \\ ${ }^{\mathrm{b}}$ OCA, Mont Gros 06304 Nice cedex, France \\ ${ }^{c}$ LESIA, Observatoire de Paris, 92195 Meudon cedex, France \\ ${ }^{\mathrm{d} J P L}, 4800$ Oak Grove Drive, MS 171-113, Pasadena, CA, USA
}

\begin{abstract}
Recent site seeing testing campaigns conducted by our team from University of Nice ${ }^{1}$ show that Dome C represents the best site on Earth for astronomical high angular resolution (HAR) observations at optical and IR wavelengths. The dramatic gain over relevant HAR parameters $r_{0}, L_{0}, \theta_{0}$ and $\tau_{0}$, added to very low temperatures during the polar winter nights $\left(-70^{\circ} \mathrm{C}\right)$, the dry atmosphere and the possibility of continuous observations during several nights make Dome $\mathrm{C}$ the ideal site for deploying a kilometric optical interferometer before the 2015 horizon. Here we describe the concept of Kiloparsec Explorer for Optical Planet Search (KEOPS) that is studied by our group at LUAN. KEOPS is an interferometric array of 36 off-axis telescopes, each $1.5 \mathrm{~m}$ in diameter. Its kilometric baselines open sub-mas snap-shot imaging possibilities to detect and characterize extra-solar planetary systems, especially exo-Earths out to 300 parsecs from the visible to the thermal IR. KEOPS can be considered as a DARWIN/TPF challenger but at a much lower cost.
\end{abstract}

Keywords: imaging interferometry, high angular resolution

\section{INTRODUCTION}

KEOPS is envisioned as a mission facility at Dome C. Its primary goal is to explore all $\mathrm{G}, \mathrm{K}$ and $\mathrm{M}$ stars out to 1 kiloparsec following a solar-systems outside our solar-system observing strategy and point of view. The collecting area of 36 telescopes of $1.5 \mathrm{~m}$ diameter makes KEOPS comparable to a $10 \mathrm{~m}$ class telescope. Operated at Dome C, KEOPS attains however the sensitivity of a 20-30m ELT from todays ground sites. The primary goal of KEOPS is the direct detection and the spectral characterization of exoplanets (ExPNs), ultimately hunting exo-Earths in habitable zones. This is done following the nulling method called IRAN $^{2}$ (Fig. 1).
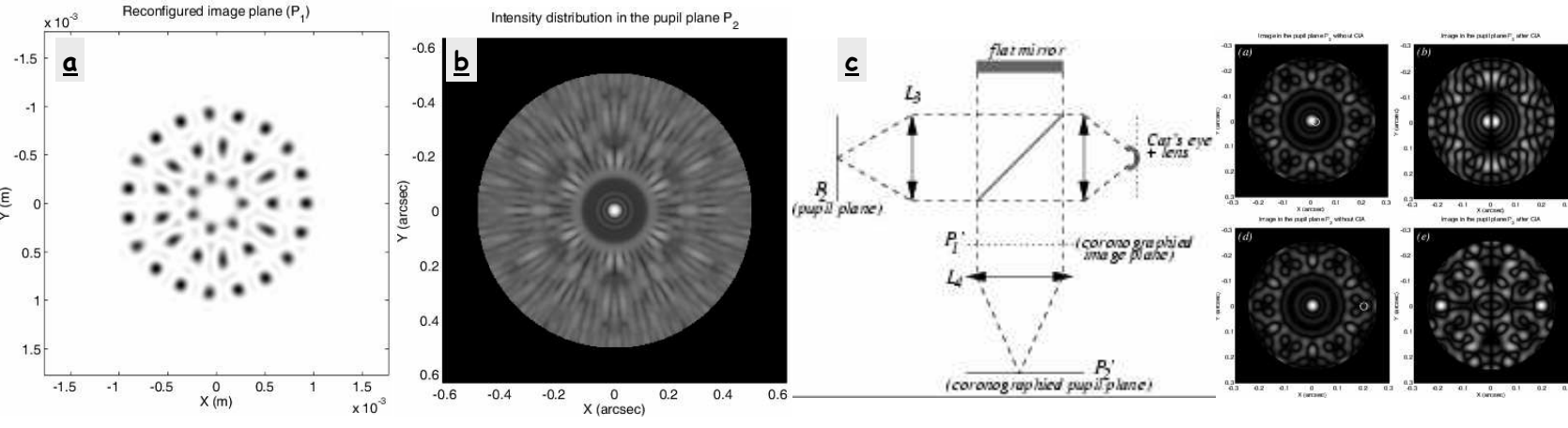

Fig. 2. IRAN concept: (a) image densification, (b) pupil plane interferometry, (c) AIC - (Achromatic InterferoCoronograph) to detect high contrast exoplanetary systems

*Farrokh.Vakili@unice.fr 
Other KEOPS targets are imagery of gravitational mirages from $\mu$-lensing events by measuring the impact parameter and derive the lens mass distribution from the temporal follow-up interferometry of these events, spatial resolution of central regions of AGNs to measure their astrometric dance due to Sne explosions, direct asterosismology of discresolved nearby stars by spatial tuning/filtering $2<1 \& \mathrm{~m}<10$ pulsationnal modes, plus imagery of stellar magnetic topology, rotational flattening $\&$ differential rotation.

Measuring the angular diameters of RSG, post-AGB and winds of WR, LBV and early-type SG in Magellanic clouds (circumpolar) and kinematics of globular clusters from narrow angle differential astrometry can also be envisioned.

\section{GENERAL FEATURES OF KEOPS}

KEOPS is a deployable array of 6,12 and 18 (final 36) 1.5m optical telescopes spread over 3 rings (Fig. 2). In its final version, the total collecting surface will be $\sim 60 \mathrm{~m}^{2}$ (equivalent to a Keck), but because of reduced atmospheric emission in the in the IR, the array will be the equivalent of a 30m ELT: we show below that working in the mid- and thermal infrared at $2-10 \mu \mathrm{m}$ ( $\mathrm{K}$ band), up to $3 \mathrm{mag}$ gain over classical sites is achieved. The radial distances of $250 \mathrm{~m}, 600 \mathrm{~m}$ and $980 \mathrm{~m}$ respectively from the central beam-collector-hub are calculated so as to optimize the array antenna beam to be a 1 mas at $10 \mu \mathrm{m}(1 \mathrm{AU}$ at $1 \mathrm{kpc})$ IRAN pseudo-Airy pattern across a FOV limited to 1". This means snap-cot direct imaging with coronographic capabilities of about $700^{2}$ resels in the FOV.

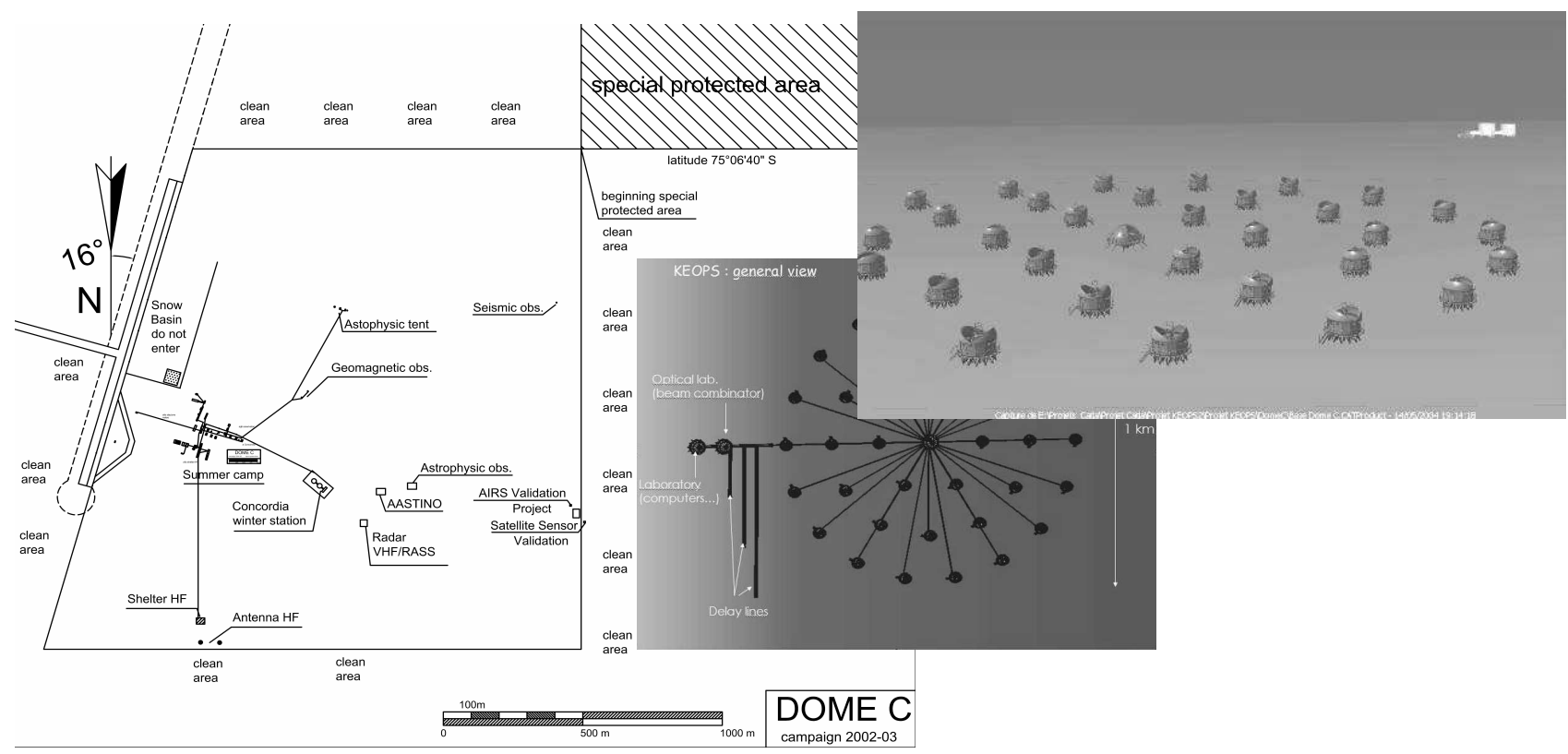

Fig. 2. Proposed layout at Dome C

The afocal beams from the Cassegrain-Coudé telescopes are sent through buried pipelines in a $10^{-2}$ vacuum minimizing horizontal propagation seeing and atmospheric dispersion effects for off-zenith sources. The 3 bundles of afocal beams from the 3 rings of telescopes are directed from the central hub towards a sideral Optical-Path-Equalizer (OPE) outside the array. The central hub, hosting the metrological laser, encloses field relay optics \& tip-tilt correction that transmit the 36 beams towards the OPE. The delay lines are disposed circularly homothetical of KEOPS array and use switchyards for minimum stroke. Secondary stage piezo delay-lines co-phase the 36 primary wavefronts. 


\section{INSTRUMENT ELEMENTS}

\subsection{Off-Axis Telescopes Optimized for IR Interferometry \& Coronography}

KEOPS primary telescopes (Fig. 3) are $1.5 \mathrm{~m}$ off-axis parabola (from master $\mathrm{F} / \mathrm{D}=1$ paraboloids) with 3 reflection Cassegrain-Coudé afocal optics and $60^{\circ}$ zenithal distance pointing. Thus KEOPS can access all sources in a $120^{\circ}$ cone from Dome C. The alt-alt mount is similar to the ESO-CAT at La Silla. It is transportable in a standard Antarctica container and can be assembled from 4 main parts: the mirror+mirror-cell, the light folding secondary+tertiary mechanics, the horse-shoe support, the horse-shoe mount itself. The optics can be passively cooled to $-60^{\circ} \mathrm{C}$ during the winter night. The absence of central obstruction minimizes both the IR background and the diffraction noise for coronagraphy in the nulling mode of KEOPS (see below). The pointing primary FOV is 1', and 20" at the central hub. Field-rotation is corrected by a K-prism.

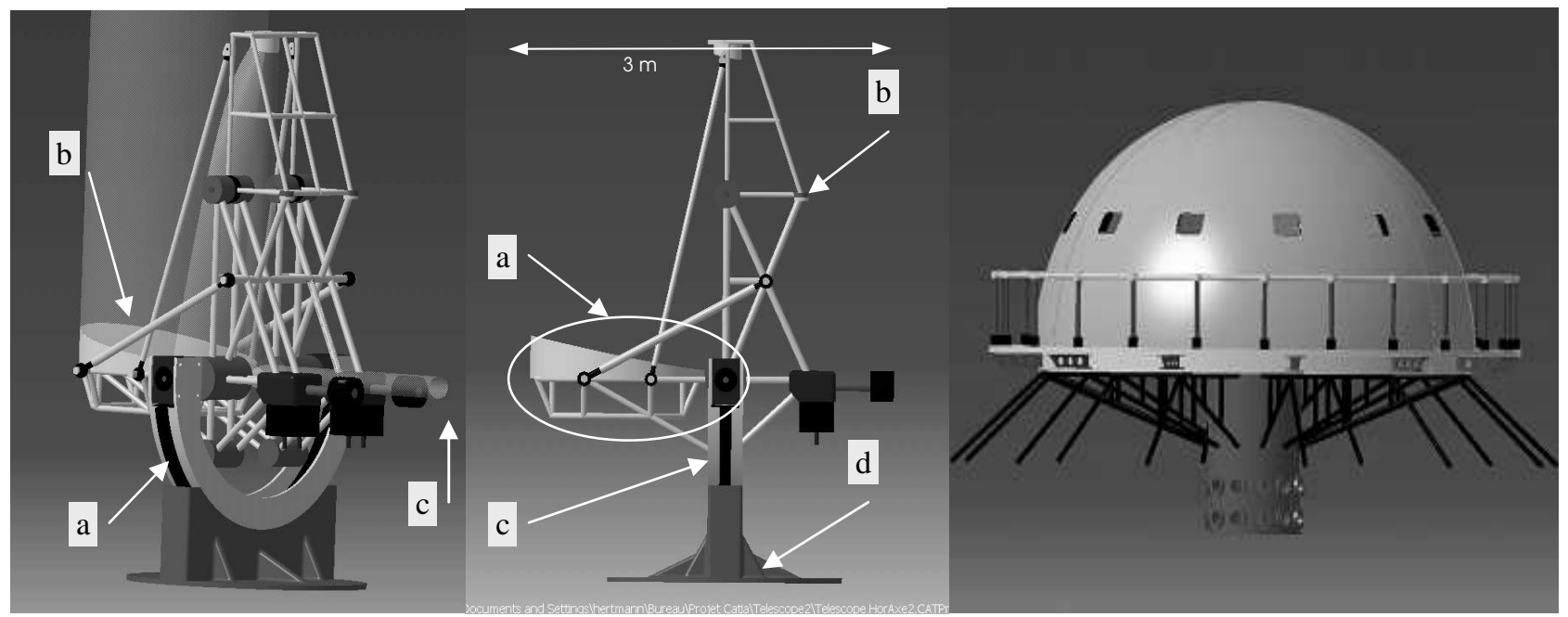

Fig. 3. Left: KEPOS telescopes Cassegrain-Coudé afocal optics: (a) Alt-alt mount similar to ESO CAT (La Silla), (b) Off axis parabola $\mathrm{d}=1 \mathrm{~m} 50 \mathrm{~F}=3 \mathrm{~m}$ (from master $\mathrm{F} / \mathrm{D}=1$ ) (avoid central obstruction and IR emission), (c) afocal output beam. Center: Easily transportable in 4 parts in standart containers, assembled on site: (a) Part 1 : mirror+support, (b) Part 2: optical tube + secondary and tertiary mirrors, (c) Part 3: horse-shoe mount, (d) Part 4: horse-shoe support. Right: central beam collector (hub)

The Central beam collector (hub) collects the 36 afocal beams from the 3 rings of telescopes, that have traveled through $10^{-2}$ vacuum optical tunnels, in order to minimize horizontal propagation seeing and atmospheric dispersion effects for off-zenith sources. The problem of the deformation of the snow surface over hectometer scales could be overcome by external service tunnels of compacted snow, the by-product of the metrology becoming a gift for the glaciologists. 


\subsection{Optical study}

P. Assus (OCA) has realized a ZEMACS study (Fig. 4) of the off-axis PSF of KEOPS individual telescopes (V band, $\mathrm{l}=550 \mathrm{~nm}$ ). The unaberrated field is approx. 2 arcmin, which is consistent with interferometry limitation where field is 1 arcsec.

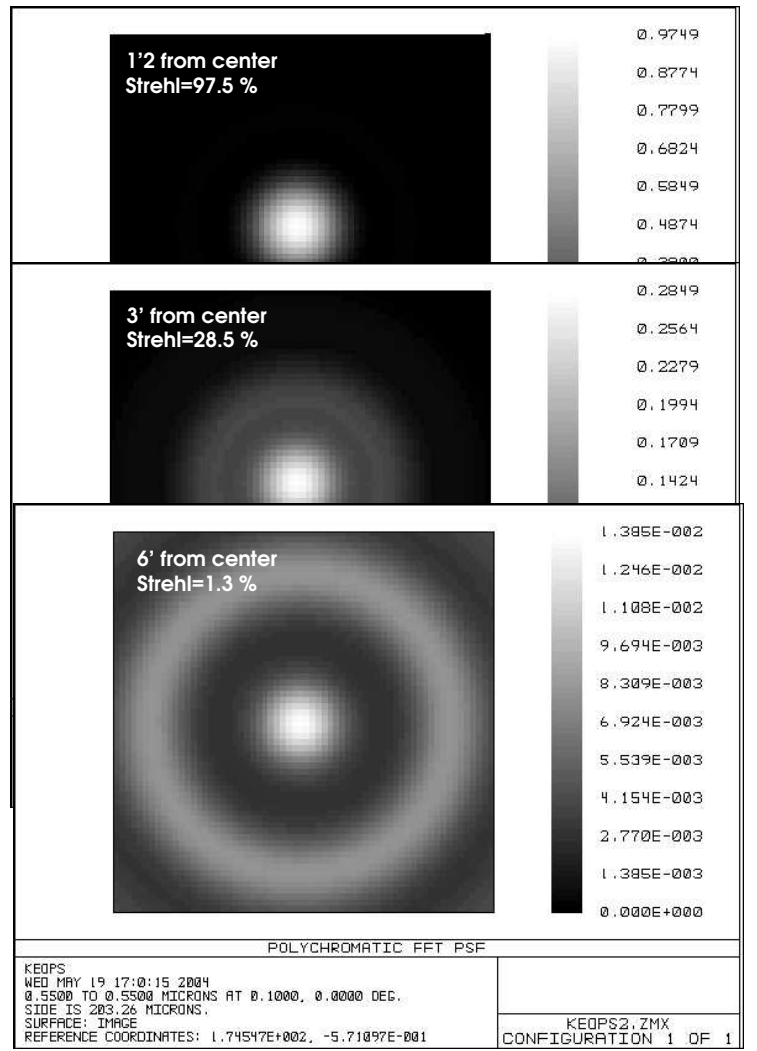

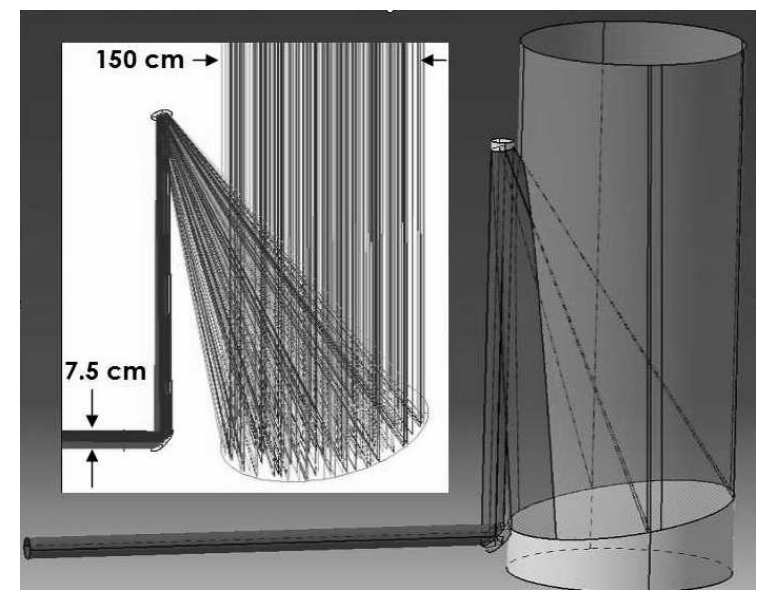

Fig. 4. Ray-tracing and the PSF for KEOPS telescopes at the level of the beam-collector central hub (V band, $1=550 \mathrm{~nm}$ ). The unaberrated field is approx. 2 arcmin, which is consistent with interferometry limitation where field is 1 arcsec.

\section{SCIENCE WITH KEOPS}

Beichman proposed ${ }^{3}$ to associate the planet detection probability to a Poisson law with the parameter $\eta N$ ( $\eta$ being the mean number of planets around a star and $N$ the number of observed stars). If the $\eta$ parameter value is 0.1 (suggested by giant planets studies) observing 30 stars should be enough to get a probability of $95 \%$ to detect one planet, 175 observed stars to detect at least 10 planets with the same probability. If the sample contains about 400 stars, we notice (Fig. 5) that an $\eta$ parameter of about 0.01 still allows the detection of at least one planet (with a probability of $95 \%$ ). We conclude that observing about 500 stars will give a good probability (95\%) to detect at least one planet if the $\eta$ parameter is almost 0.01 and more than 10 planets if the $\eta$ parameter is almost 0.1 . 


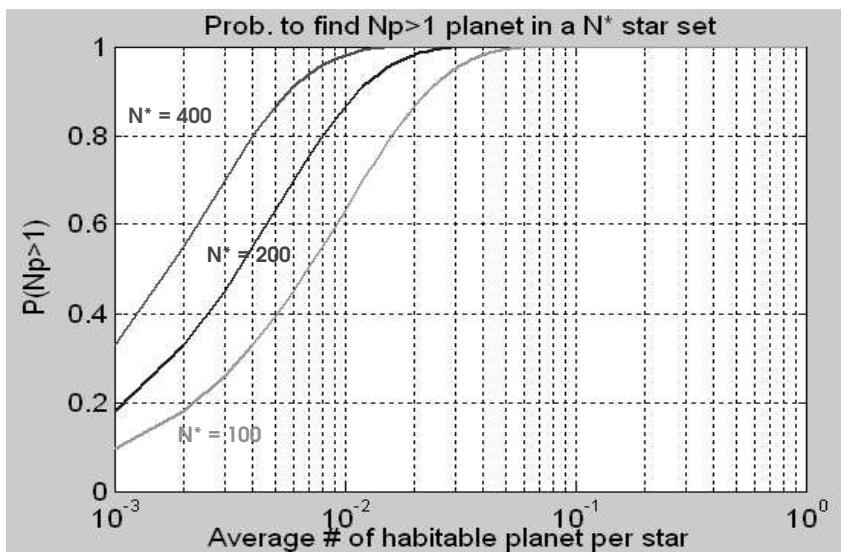

Fig. 5. Computed probability of finding at least 1 planet versus unknown average number of habitable planets per star. $\mathrm{N}$ is the total number of planets screened.

According to the CDS, there are about $300 \mathrm{G}, 30 \mathrm{~K}$ and $30 \mathrm{M}$ sub-giant, main-sequence and sub-dwarf stars in the Dome C sky (at less than $60^{\circ}$ away from the south pole's zenith; $45^{\circ}$ because of KEOPS possibilities of orientation + $15^{\circ}$ because Dome $\mathrm{C}$ is $15^{\circ}$ away from south pole). Those stars are up to 300pc (M), 60pc (G) and 20pc (K) away from the

Earth. If we assume the stars repartition constant up to 300pc away from the sun, we have densities of $5.6310^{-6}$,

$1.0110^{-4}$ and $2.8110^{-3}$ for respectively G, K and M stars. KEOPS will be sized to get an observable sky including 500 stars in theses conditions.

\subsection{KEOPS observable sky}

The KEOPS observable sky shape is obtained by considering a volume limited by a maximum zenith angle of $45^{\circ}$. KEOPS is an interferometer and its resolution allows it to see up to a distance decreasing with the zenith angle so that the volume is also limited by a depth $d$ that decreases with the zenith distance $\left(d=d_{0} \cos z\right)$. We also have to take account of the Earth rotation that moves the described volume around the Earth's rotation axis

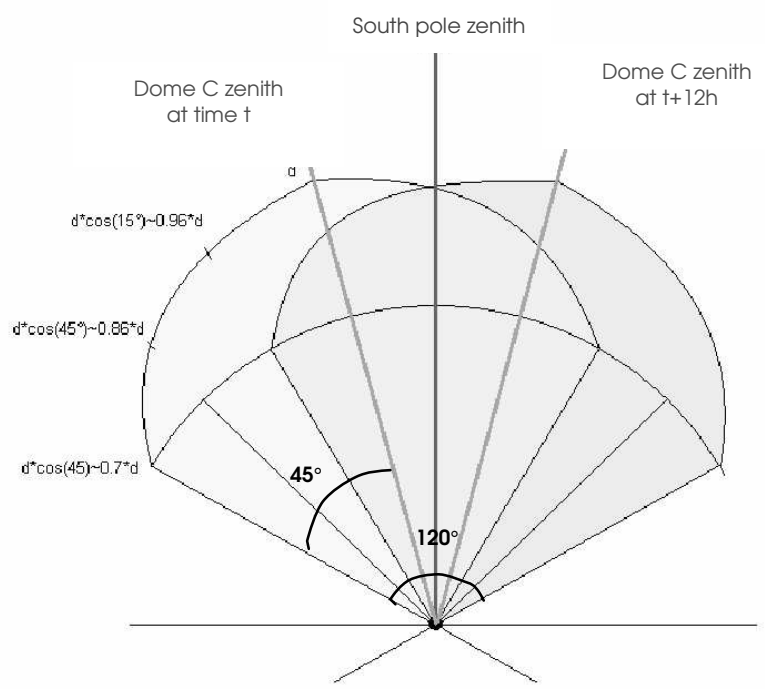

Fig. 6. KEOPS observable sky 
So KEOPS can access a volume limited by a zenith angle of $60^{\circ}$ from the South Pole. At a given time, this volume is not fully reachable but is entirely covered in 1 day (Earth rotation period, see Fig. 6). The volume includes a constant observable part limited by a zenith angle of $30^{\circ}$.

We calculated that to observe possible planets in the habitable zone of 500 stars KEOPS must be able to see G stars up to $254 \mathrm{pc}$, K stars up to $161 \mathrm{pc}$ and $\mathrm{M}$ stars up to $70 \mathrm{pc}$ for the $10 \mu \mathrm{m}$ band. It must be able to observe $\mathrm{G}$ stars up to $160 \mathrm{pc}$, $\mathrm{K}$ stars up to $128 \mathrm{pc}$ and $\mathrm{M}$ stars up to $76 \mathrm{pc}$ in the $2 \mu \mathrm{m}$ band. Those numbers are taking account of the habitable zone position that is function of the star's temperature and the contrast ratio that is function of the wavelength but also of the star temperature.

The coronograph's extinction ratio should be about $3.710^{5}$ in the $10 \mu \mathrm{m}$ band and $210^{7}$ in the $2 \mu \mathrm{m}$ band.

\subsection{Exposure time}

At $10 \mu \mathrm{m}$, the exposure time is mainly increased by the atmosphere's background emission and the instrument thermal emission. Since we attempt to detect an Earth-like planet in the habitable zone, its emission spectrum is close to Earth's own atmosphere and the one of the instrument. A first way to reduce the integration time it is to go to Dome $\mathrm{C}\left(-70^{\circ} \mathrm{C}\right)$; there, the exposure time is more than 10 times shorter than in temperate sites $\left(0^{\circ} \mathrm{C}\right)$. A second way to reduce the exposure time is to use the $2 \mu \mathrm{m}$ band (Fig. 7).

The exposure time needed to detect a planet located at $25 \mathrm{pc}$, with a signal to noise ratio of 5 is 100 days in the $10 \mu \mathrm{m}$ band. It falls to less than 6 hours in the $2 \mu \mathrm{m}$ band for a planet hosted by a $\mathrm{G}$ star and less than 1 hour for a planet hosted by a $\mathrm{M}$ star (because of thermal background and lower instrument emission level).

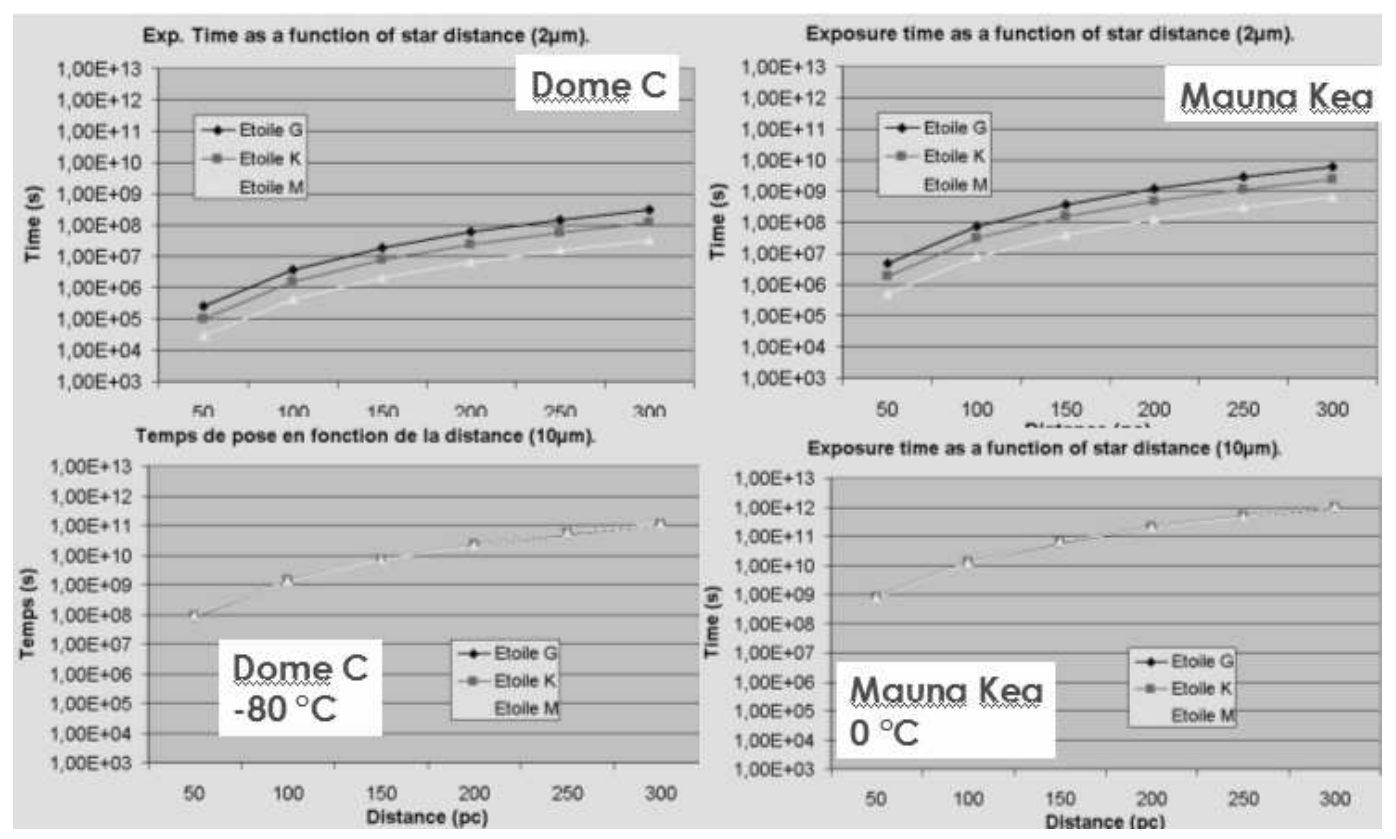

Fig. 7. Exposure time as a function of distance

For the very far planet observations, the exposure time rise $10^{8} \mathrm{~s}$ at $50 \mathrm{pc}$ and $10^{11} \mathrm{~s}$ at $300 \mathrm{pc}$ in the $10 \mu \mathrm{m}$ band. In the $2 \mu \mathrm{m}$ band, it is around $10^{5} \mathrm{~s}$ at $50 \mathrm{pc}$ and $10^{8} \mathrm{~s}$ at $300 \mathrm{pc}$. 
Overall, the $10 \mu \mathrm{m}$ band looks unusable for the observation of far, faint objects. KEOPS will therefore work in the $2 \mu \mathrm{m}$ band.

In a 6 hours exposure time, its sensibility allows KEOPS to observe far enough to access about 100 stars and to detect at least on planet with a $99.9 \%$ probability if $\eta=0.1$, and with a probability of $63 \%$ if $\eta=0.01$. In 12 hours, KEOPS scans 250 stars in its observation volume that will allow to ensure the detection of at least 1 planet with a $99.9 \%$ probability if $\eta=0.1$ and with a probability of $91.8 \%$ if $\eta=0.01$. In 1 day exposure time, KEOPS will extend its observation volume enough to include 350 stars and ensure the detection of at least 1 planet with a probability of $99.9 \%(\eta=0.1)$ or a probability of $97 \%(\eta=0.01)$.

\subsection{Other science targets for KEOPS}

Direct snap-shot imaging capabilities make KEOPS suitable for stellar surface imaging. At $2.2 \mu \mathrm{m}$ a 0.4 mas resolution would yield $25^{2}$ resels on $\alpha$ Cen.

Another opportunity would be direct microlensing imaging, as proposed by Delplancke ${ }^{4}$.

\section{DEVELOPEMENT TIME SCALE AND CONCLUSIONS}

The KEOPS development can be split in 6 steps from 0 to 5. The 0 step (the current one) consists in R\&D studies and is planned to last until 2006. Among the challenges: telescopes, beam stabilisation, cophasing, delay lines, recombiner, coronograph, science. The step 1 will be the single telescope operations and will happen during 2007. This is a testbench and science operation phase, including planeto/asterosismology (Schmider JISCO/Mosser SIAMOIS) and $\mu$ lensing as proposed by Ragazzoni. Next, the step 2 will make two telescopes working in interferometer mode (20082009). This could be a result of a synergy with the APISD project ${ }^{5}$. The next step will deploy the first KEOPS ring composed of 6 telescopes, and here a connection with the API project can be envisioned ${ }^{6}$. Finally, the KEOPS project will be ready with its 36 telescopes around 2015 .

\section{REFERENCES}

1. Aristidi et al, "Antarctic site testing: First daytime seeing monitoring at Dome C", $A \& A, 4$ 406, L19-L22, 2003, see also http://www-astro.unice.fr/Concordiastro/indexantartic.html.

2. Aristidi et al, these proceedings.

3. Beichman, "How many stars are enough?", Proceedings of the Conference on Towards Other Earths: DARWIN/TPF and the Search for Extrasolar Terrestrial Planets, M. Fridlund, T. Henning, 271-275, ESA, Heidelberg, 2003.

4. Delplancke et al, "Resolving gravitational microlensing events with long-baseline optical interferometry. Prospects for the ESO Very Large Telescope Interferometer", $A \& A, \mathbf{3 7 5}, 701-710,2001$

5. Coudé du Foresto et al, "Interferometric spectrophotometry of Pegasides: APISD the Antarctic Plateau Interferometer Science Demonstrator", Memorie della Societa Astronomica Italiana Supplement, 2, 212, 2003

6. Swain et al, "The Antarctic Planet Interferometer", American Astronomical Society Meeting 203, \#38.15, 2003 